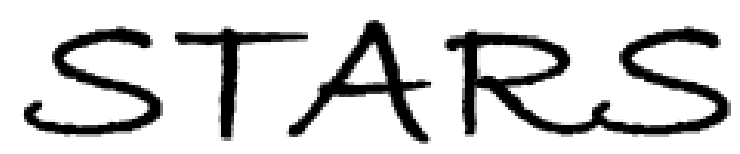

University of Central Florida

STARS

\title{
Impact of wireless channel uncertainty upon M-ary distributed detection systems.
}

Zahra Hajibabaei Najafabadi

University of Central Florida

Part of the Electrical and Electronics Commons

Find similar works at: https://stars.library.ucf.edu/etd

University of Central Florida Libraries http://library.ucf.edu

This Masters Thesis (Open Access) is brought to you for free and open access by STARS. It has been accepted for inclusion in Electronic Theses and Dissertations, 2004-2019 by an authorized administrator of STARS. For more information, please contact STARS@ucf.edu.

\section{STARS Citation}

Hajibabaei Najafabadi, Zahra, "Impact of wireless channel uncertainty upon M-ary distributed detection systems." (2016). Electronic Theses and Dissertations, 2004-2019. 4901.

https://stars.library.ucf.edu/etd/4901

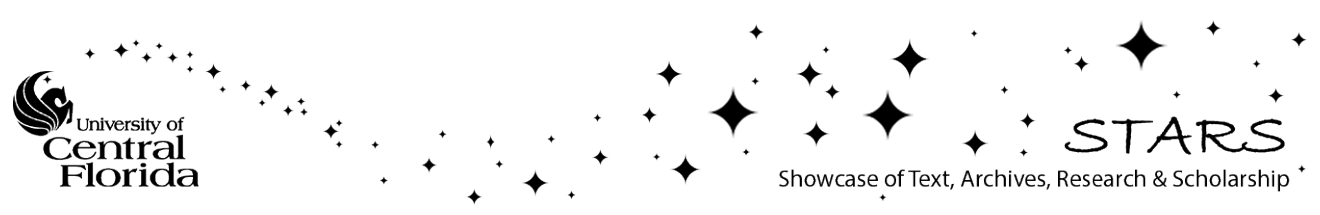




\title{
IMPACT OF WIRELESS CHANNEL UNCERTAINTY UPON M-ARY DISTRIBUTED DETECTION SYSTEMS
}

by

\section{ZAHRA HAJIBABAEI}

M.Sc. Isfahan University of Technology, 2012

\begin{abstract}
A thesis submitted in partial fulfilment of the requirements for the degree of Master of Science in the Department of Electrical and Computer Engineering in the College of Engineering and Computer Science at the University of Central Florida, Orlando, Florida
\end{abstract}

Spring Term

2016 
(c) 2016 Zahra Hajibabaei 


\begin{abstract}
We consider a wireless sensor network (WSN), consisting of several sensors and a fusion center (FC), which is tasked with solving an $M$-ary hypothesis testing problem. Sensors make $M$-ary decisions and transmit their digitally modulated decisions over orthogonal channels, which are subject to Rayleigh fading and noise, to the FC. Adopting Bayesian optimality criterion, we consider training and non-training based distributed detection systems and investigate the effect of imperfect channel state information (CSI) on the optimal maximum a posteriori probability (MAP) fusion rules and detection performance, when the sum of training and data symbol transmit powers is fixed. Our results show that for Rayleigh fading channel, when sensors employ $M$-FSK or binary FSK (BFSK) modulation, the error probability is minimized when training symbol transmit power is zero (regardless of the reception mode at the FC). However, for coherent reception, $M$-PSK and binary PSK (BPSK) modulation the error probability is minimized when half of transmit power is allocated for training symbol. If the channel is Rician fading, regardless of the modulation, the error probability is minimized when training transmit power is zero.
\end{abstract}




\section{TABLE OF CONTENTS}

LIST OF FIGURES . . . . . . . . . . . . . . . . . . . . . vi

CHAPTER 1: INTRODUCTION . . . . . . . . . . . . . . . . 1

CHAPTER 2: OPTIMAL FUSION RULE FOR M-ARY DISTRIBUTED DETECTION WITH M-ARY MODULATION . . . . . . . . . . . . . . . . . 4

System Model and Problem Statement . . . . . . . . . . . . . . . . . . . 4

Coherent Reception with $M$-PSK and $M$-FSK $\ldots \ldots \ldots \ldots$

Complex Channel Estimation . . . . . . . . . . . . . . . . . . . 8

Optimal Fusion Rule Corresponding to $M$-PSK . . . . . . . . . . . . 8

Optimal Fusion Rule Corresponding to $M$-FSK . . . . . . . . . . . . . . . 9

Non Coherent Reception with $M$-FSK . . . . . . . . . . . . . . . . 10

Channel Amplitude Estimation . . . . . . . . . . . . . . . . . 10

Optimal Fusion Rule with Channel Amplitude Estimation . . . . . . . . . . . . . 10

Optimal Fusion Rule without Channel Amplitude Estimation . . . . . . . . . . . . 12

CHAPTER 3: OPTIMAL FUSION RULE FOR M-ARY DISTRIBUTED DETECTION WITH BINARY MODULATION . . . . . . . . . . . . . . . . . 13 
System Model and Problem Statement . . . . . . . . . . . . . . . . . . . . . 13

Coherent Reception with BPSK and BFSK . . . . . . . . . . . . . 16

Complex Channel Estimation . . . . . . . . . . . . . . . . . 16

Optimal Fusion Rule Corresponding to BPSK . . . . . . . . . . . . . . . 16

Optimal Fusion Rule Corresponding to BFSK . . . . . . . . . . . . . . . . 17

CHAPTER 4: NUMERICAL RESULTS . . . . . . . . . . . . . . . . . . 19

CHAPTER 5: CONCLUSIONS . . . . . . . . . . . . . . . . 25

LIST OF REFERENCES . . . . . . . . . . . . . . . . . . . . . . . . 26 


\section{LIST OF FIGURES}

Figure 4.1: Training based coherent reception with 4-PSK and 4-FSK modulation: (a) $P_{e}$ vs. SNR, (b) $P_{e}$ vs. $r_{d} \ldots \ldots \ldots \ldots \ldots \ldots$

Figure 4.2: Noncoherent reception with 4-FSK modulation: (a) $P_{e}$ vs. SNR for training and non-training based, (b) $P_{e}$ vs. $r_{d}$ for training based. . . . . . . . . . 22

Figure 4.3: Rayleigh fading channel model with BPSK and BFSK modulation: (a) $P_{e}$ vs. SNR, (b) $P_{e}$ vs. $r_{d} \ldots \ldots \ldots \ldots \ldots \ldots \ldots$

Figure 4.4: Rician fading channel model with BPSK and BFSK modulation: (a) $P_{e}$ vs. $\mathrm{SNR},(\mathrm{b}) P_{e}$ vs. $r_{d} \ldots \ldots \ldots \ldots \ldots \ldots$ 


\section{CHAPTER 1: INTRODUCTION}

In recent years wireless sensor networks (WSN) have received great attention in both theory and application. The applications span from environmental monitoring to monitoring of manufacturing processes, robotics and medical applications. One of prevalent applications is monitoring or detecting a physical phenomenon or environmental condition by means of geographically distributed battery-powered sensors deployed over a sensing field. In signal processing society, the problems associated with detecting or estimating the phenomenon, employing multiple observations coming from distributed sensors are usually termed distributed detection [1,2], or distributed estimation [3-9], respectively.

Distributed detection theory is concerned with optimal designs of fusion rule at the FC and local detection rules at the sensors (local detectors), such that a predetermined detection performance criterion is optimized. We consider a wireless sensor network, consisting of a set of spatially distributed sensors and a FC, that is tasked with solving an $M$-ary distributed detection problem. In particular, we consider the problem of distributed classification of $M$ independent Gaussian sources with identical variances and different means. We assume each sensor processes locally its observation to form a local decision and transmits its digitally modulated decision to the FC, over a fading channel. The FC is tasked with fusing all the received signals from the sensors directly, via applying the optimal fusion rule, and making the final decision.

Channel-aware binary distributed detection for fusion of binary decisions transmitted over fading channels was first discussed in [10], where the FC fuses the received signals from the sensors directly (without demodulating the transmitted symbols). The works on channel-aware binary distributed detection are mainly built on the assumption that perfect knowledge of phase or amplitude of the fading channel coefficients are available at the FC [11-17]. Today's wireless communication systems with coherent reception rely upon training in order to facilitate channel estimation at the 
receiver. In fact, quantifying the effect of imperfect channel state information (CSI) and channel estimation error on the design and performance of wireless communication systems is a challenging problem, that has attracted the attention of researchers over the past decade $[18,19]$. Recently, channel-aware binary distributed detection with imperfect CSI was studied in [20-22]. Compared with binary distributed detection, the literature on channel-aware $M$-ary distributed detection falls short [23-26], where the communication channels are modeled as additive white Gaussian noise (AWGN) [23] and Rayleigh fading with perfect CSI available at the FC [24,25]. To the best of our knowledge, this is the first work that considers the impact of imperfect CSI on the design and performance of channel-aware $M$-ary distributed detection systems.

In this work, we address the following questions: how are the optimal fusion rules affected by channel estimation error? how can we mitigate the negative impact of channel estimation error via optimizing transmit power allocation between data and training symbols? how do the answers to the above questions change as the reception mode at the FC and modulation scheme at the sensors vary? For non coherent reception, how do the optimal fusion rules differ for training and non-training based systems, where the sensors do not transmit training symbols (for estimating channel amplitudes) and the FC only relies on the knowledge of the channel statistics? To answer these questions we consider the following cases: assuming Rician or Rayleigh block fading channel model: ( $i$ ) the FC is equipped with a coherent receiver and a training based channel estimator, sensors employ $M$-PSK or $M$-FSK modulation for transmitting their data and training symbols, (ii) the FC is equipped with a non coherent receiver and a training based channel amplitude estimator, the sensors employ $M$-FSK modulation for transmitting their data and training symbols, (iii) the FC is equipped with a non coherent receiver without a channel estimator (the FC only has the channel statistics), the sensors employ $M$-FSK modulation for transmitting their data symbols. The organization of the thesis follows: In Chapter 2, we derive the optimal fusion rules for cases (i), (ii), (iii) explained above when sensors use $M$-ary modulation. In chapter 3 , we derive optimal fusion rule for coherent reception when we apply BFSK and BPSK modulations. Chapter 4, we 
compare the performance of different systems considering error probability. Chapter 5 includes concluding remarks.

Notation: Boldface lower case letters without and with underline denote random scalars and random vectors, respectively. Boldface upper case letters denote matrices. 


\section{CHAPTER 2: OPTIMAL FUSION RULE FOR M-ARY DISTRIBUTED DETECTION WITH M-ARY MODULATION}

In this chapter, we consider an $M$-ary distributed detection system, in which sensors send their modulated decisions over orthogonal noisy channels with Rayleigh fading channel model to a FC. We use M-PSK or M-FSK for sending data from sensors to FC. We consider training and nontraining based systems and investigate the effect of imperfect channel state information on the fusion rules and detection performance, assuming the sum of training and data symbol transmit powers is fixed. This chapter is organized as follows. First, we present our system model. Next for random and deterministic channel models, we derive the optimal fusion rules and the special case of $M=2$.

\section{System Model and Problem Statement}

We consider the problem of testing which of the $M \geq 2$ hypotheses $\left\{H_{j}\right\}_{j=1}^{M}$ has been occurred, assuming $\pi_{j}$ is the a priori probability of $H_{j}$ happening. Our system consists of a FC and $N$ spatially distributed sensors, which is tasked with solving this $M$-ary hypothesis testing problem. Let $\boldsymbol{x}_{k}$ denote the local observation collected at sensor $k$ during an observation period. We assume that $\boldsymbol{x}_{k}$ 's are independent across sensors, conditioned on a particular hypothesis, i.e., $f\left(\boldsymbol{x}_{1}, \boldsymbol{x}_{2}, \ldots, \boldsymbol{x}_{N} \mid H_{j}\right)=\prod_{k=1}^{N} f\left(\boldsymbol{x}_{k} \mid H_{j}\right)$ for $j=1, \ldots, M$, where $f($.$) is the probability density$ function (pdf). Suppose $\boldsymbol{x}_{k}$ at sensor $k$ under hypothesis $H_{j}$ is

$$
H_{j}: \boldsymbol{x}_{k}=\boldsymbol{z}_{j}+\boldsymbol{n}_{k}, \quad j=1, \ldots, M
$$


where $\boldsymbol{z}_{j}$ 's are Gaussian signal sources with different means and equal variances, i.e., $\boldsymbol{z}_{j} \sim$ $N\left(m_{j}, \sigma_{z}^{2}\right), \boldsymbol{n}_{k}$ 's are Gaussian measurement noises $\boldsymbol{n}_{k} \sim N\left(0, \sigma_{n}^{2}\right)$, and $\boldsymbol{z}_{j}, \boldsymbol{n}_{k}$ are all mutually uncorrelated. Each sensor applies a local rule to decide which of the $M$ hypotheses has occurred, such that the error probability at the sensor is minimized, i.e., the local detector of sensor $k$ finds $l_{k}=\arg \min _{j}\left|\boldsymbol{x}_{k}-m_{j}\right|$ and decides hypothesis $H_{l_{k}}$. Let $p_{i j}^{k}$ denote the probability that sensor $k$ decides on $H_{i}$, given that the true hypothesis is $H_{j}$. For the sensing model in (2.1), one can verify that $p_{i j}^{k}=p_{i j}$ given below

$$
p_{i j}= \begin{cases}Q\left(\frac{m_{i}+m_{i-1}-2 m_{j}}{2\left(\sigma_{n}^{2}+\sigma_{z}^{2}\right)}\right)-Q\left(\frac{m_{i}+m_{i+1}-2 m_{j}}{2\left(\sigma_{n}^{2}+\sigma_{z}^{2}\right)}\right) & i \neq 1, M \\ 1-Q\left(\frac{m_{1}+m_{2}-2 m_{j}}{2\left(\sigma_{n}^{2}+\sigma_{z}^{2}\right)}\right) & i=1 \\ Q\left(\frac{m_{M}+m_{M-1}-2 m_{j}}{2\left(\sigma_{n}^{2}+\sigma_{z}^{2}\right)}\right) & m=M\end{cases}
$$

where we define the $Q$-function as $Q(x)=\frac{1}{\sqrt{2 \pi}} \int_{x}^{\infty} e^{-t^{2} / 2} d t$. Sensor $k$ employs an $M$-ary digital modulator to map its $M$-ary decision to a symbol and transmits this symbol with power $P_{d}$. In this chapter, we consider $M$-PSK and $M$-FSK modulation at the sensors. Let $\boldsymbol{u}_{k}$ and $\underline{\boldsymbol{u}}_{k}$ denote the modulated symbol at sensor $k$ corresponding to $M$-PSK and $M$-FSK modulation, respectively, where $\boldsymbol{u}_{k} \in\left\{e^{j 2 \pi \frac{i-1}{M}}, i=1, \ldots, M\right\}, \underline{\boldsymbol{u}}_{k} \in\left\{\underline{e}_{i}, i=1, \ldots, M\right\}$ and $\underline{e}_{i}$ is an $1 \times M$ canonical vector whose all elements except the $i$-th one are zeros. We refer to the modulated symbols $\boldsymbol{u}_{k}, \underline{\boldsymbol{u}}_{k}$ as data symbols and $P_{d}$ as data symbol transmit power. Assuming the data symbols are sent over orthogonal channels between sensors and the FC, the channel output corresponding to sensor $k$ at the FC upon the reception of data symbol is

$$
\begin{aligned}
& \boldsymbol{y}_{d k}=\sqrt{P_{d k}} \boldsymbol{h}_{k} \boldsymbol{u}_{k}+\boldsymbol{n}_{d k} \text { M-PSK, } \\
& \underline{\boldsymbol{y}}_{d k}=\sqrt{P_{d k}} \boldsymbol{h}_{k} \underline{\boldsymbol{u}}_{k}+\underline{\boldsymbol{n}}_{d k} \text { M-FSK, }
\end{aligned}
$$


where $P_{d k}=G P_{d}\left(\Delta_{k}\right)^{-\epsilon}$ is the received power corresponding to the data symbol, $G$ is a constant, $\epsilon$ is the path loss exponent, and $\Delta_{k}$ is the distance between sensor $k$ and the FC. The communication channel noises, denoted as $\boldsymbol{n}_{d k}$ and $\underline{\boldsymbol{n}}_{d k}$, are zero mean complex Gaussian $\boldsymbol{n}_{d k} \sim C N\left(0, \sigma_{n}^{2}\right)$, $\underline{\boldsymbol{n}}_{d k} \sim C N\left(0, \sigma_{n}^{2} I\right)$, where $I$ is an $M \times M$ identity matrix. We assume that the channel outputs conditioned on the channel inputs, are independent across the sensors. The complex channel coefficient $\boldsymbol{h}_{k}$ in (2.3) is modeled as $\boldsymbol{h}_{k} \sim C N(0,1)$ and is represented as $\boldsymbol{h}_{k}=\boldsymbol{\alpha}_{k} e^{j \phi_{k}}$, where the amplitude $\boldsymbol{\alpha}_{k}$ and the phase $\phi_{k}$, respectively, have Rayleigh and uniform distributions.

To enable training based channel estimation, we assume that the channel coefficients are fixed for two consecutive symbol intervals, and each sensor sends a training symbol with power $P_{t}$ along with its data symbol. We refer to the symbols $u_{t}, \underline{u}_{t}$ as training symbols and $P_{t}$ as training symbol transmit power. Without loss of generality, we assume $u_{t}=1$ and $\underline{u}_{t}=\underline{e}_{1}$, respectively, when the sensors employ $M$-PSK and $M$-FSK schemes. Training symbols are also sent over orthogonal channels between sensors and the FC, prior to sending data symbols. The channel output corresponding to sensor $k$ at the $\mathrm{FC}$ upon the reception of training symbol is

$$
\begin{array}{ll}
\boldsymbol{y}_{t k}=\sqrt{P_{t k}} \boldsymbol{h}_{k} u_{t}+\boldsymbol{n}_{t k} & \text { M-PSK, } \\
\underline{\boldsymbol{y}}_{t k}=\sqrt{P_{t k}} \boldsymbol{h}_{k} \underline{u}_{t}+\underline{\boldsymbol{n}}_{t k} & \text { M-FSK, }
\end{array}
$$

where the noises $\boldsymbol{n}_{t k} \sim C N\left(0, \sigma_{n}^{2}\right), \underline{\boldsymbol{n}}_{t k} \sim C N\left(0, \sigma_{n}^{2} I\right)$ are independent from $\boldsymbol{n}_{d k}$ and $\underline{\boldsymbol{n}}_{d k}$ in (2.4). Also, $P_{t k}=G P_{t}\left(\Delta_{k}\right)^{-\epsilon}$ is the received power corresponding to the training symbol. In this chapter, we consider both coherent and non coherent receivers. The unknown channel parameters to be estimated depend on the receiver structure. For a coherent receiver with a training based channel estimator and a non coherent receiver with a training based channel amplitude estimator, the unknown parameters are $\boldsymbol{h}_{k}$ and $\boldsymbol{\alpha}_{k}$, respectively. We model these as $\boldsymbol{h}_{k}=\hat{\boldsymbol{h}}_{k}+\tilde{\boldsymbol{h}}_{k}$ and $\boldsymbol{\alpha}_{k}=\hat{\boldsymbol{\alpha}}_{k}+\tilde{\boldsymbol{\alpha}}_{k}$, where $\hat{\boldsymbol{h}}_{k}$ and $\hat{\boldsymbol{\alpha}}_{k}$ are the estimates based on $\boldsymbol{y}_{t k}$ and $\boldsymbol{v}_{t k}=\left|\boldsymbol{y}_{t k}^{1}\right|^{2}$ in (2.4) 
respectively, and $\tilde{\boldsymbol{h}}_{k}$ and $\tilde{\boldsymbol{\alpha}}_{k}$ are the estimation errors ${ }^{1}$. To include the cost of channel estimation, we assume that the transmit power consumption per decision $P_{t}+P_{d}=P$ is constant. We define $P_{k}=G P\left(\Delta_{k}\right)^{-\epsilon}$ and let $r_{d}=P_{d} / P=P_{d k} /\left(P_{d k}+P_{t k}\right)=P_{d k} / P_{k}$, where $r_{d} \in[0,1]$ is the fraction of the power assigned to the data symbol.

We adopt the Bayesian criterion to find the optimal fusion rule at the FC, in order to make a global decision $u_{0} \in\left\{H_{1}, H_{2}, . ., H_{M}\right\}$. The optimal fusion rule is $u_{0}=\arg \max _{m} \pi_{m} \Theta_{m}$ where $\Theta_{m}$ varies, depending on the receiver structure and the modulation scheme. Since the channel outputs are independent across sensors, we find

$$
\Theta_{m}=\prod_{k=1}^{N} f\left(\boldsymbol{y}_{d k} \mid H_{m}\right)=\prod_{k=1}^{N} \sum_{i=1}^{M} p_{i m} f\left(\boldsymbol{y}_{d k} \mid \boldsymbol{u}_{k}(i), \hat{\boldsymbol{h}}_{k}\right)
$$

when sensors employ $M$-PSK and the receiver is coherent. When sensors employ $M$-FSK we obtain

$$
\Theta_{m}=\prod_{k=1}^{N} \sum_{i=1}^{M} p_{i m} f\left(\underline{\boldsymbol{y}}_{d k} \mid \underline{\boldsymbol{u}}_{k}(i), \boldsymbol{g}_{k}\right)
$$

where $\boldsymbol{g}_{k}=\hat{\boldsymbol{h}}_{k}$ for coherent receiver, $\boldsymbol{g}_{k}=\hat{\boldsymbol{\alpha}}_{k}$ for non coherent receiver with a training based channel amplitude estimator, and $\boldsymbol{g}_{k}$ is null for non coherent receiver without a channel estimator. Also, $\boldsymbol{u}_{k}(i), \underline{\boldsymbol{u}}_{k}(i)$ in (2.5), (2.6) are the transmitted data symbols of sensor $k$ corresponding to the decision of $H_{i}$ and $p_{i m}$ is obtained from (2.2). Since the sum of transmit powers of training and data symbols is fixed, the estimation error and thus the performance of the optimal fusion rules would depend on $P_{k}$ and the ratio $r_{d}$.

\footnotetext{
${ }^{1}$ As we mentioned, the sensors employ $M$-FSK modulation when the FC is equipped with a non coherent receiver and a training based channel amplitude estimator. Since $\underline{u}_{t}=\underline{e}_{1}$, the estimator only employs the first entry of vector $\underline{\boldsymbol{y}}_{t k}$, denoted as $\boldsymbol{y}_{t k}^{1}$, for channel amplitude estimation.
} 


\section{Coherent Reception with $M$-PSK and $M$-FSK}

\section{Complex Channel Estimation}

For the linear signal model in (2.4), the minimum mean square error (MMSE) channel estimation of $\boldsymbol{h}_{k}$ given $\boldsymbol{y}_{t k}$ is $\hat{\boldsymbol{h}}_{k}=E\left\{\boldsymbol{h}_{k} \mid \boldsymbol{y}_{t k}\right\}=\frac{\sigma_{h}^{2} \sqrt{P_{t k}}}{\sigma_{h}^{2} P_{t k}+\sigma_{n}^{2}} \boldsymbol{y}_{t k}$. substituting $\hat{\boldsymbol{h}}_{k}$ in $\boldsymbol{y}_{d k}$ in (2.3), we find:

$$
\boldsymbol{y}_{d k}=\sqrt{P_{d k}} \hat{\boldsymbol{h}}_{k} \boldsymbol{u}_{k}+\boldsymbol{w}_{k}, \text { where } \boldsymbol{w}_{k}=\sqrt{P_{d k}} \tilde{\boldsymbol{h}}_{k} \boldsymbol{u}_{k}+\boldsymbol{n}_{d k}
$$

The complex noise $\boldsymbol{w}_{k}$ in (2.7) combines the AWGN noise and the channel estimation error. Considering (2.4), we observe that $\boldsymbol{y}_{t k} \sim C N\left(0, P_{t k} \sigma_{h}^{2}+\sigma_{n}^{2}\right)$. Let $\gamma_{t k}=P_{t k} / \sigma_{n}^{2}$. Since $\hat{\boldsymbol{h}}_{k}$ is a linear function of $\boldsymbol{y}_{t k}$, we have $\hat{\boldsymbol{h}}_{k} \sim C N\left(0, \frac{\sigma_{h}^{4} \gamma_{t k}}{1+\sigma_{h}^{2} \gamma_{t k}}\right)$ and $\tilde{\boldsymbol{h}}_{k} \sim C N\left(0, \frac{\sigma_{h}^{2}}{1+\sigma_{h}^{2} \gamma_{t k}}\right)$ [20].

\section{Optimal Fusion Rule Corresponding to M-PSK}

To find $f\left(\boldsymbol{y}_{d k} \mid \boldsymbol{u}_{k}(i), \hat{\boldsymbol{h}}_{k}\right)$ in (2.5), we realize that given $\boldsymbol{u}_{k}$ and $\hat{\boldsymbol{h}}_{k}$, we have $\boldsymbol{y}_{d k} \sim$ $C N\left(\sqrt{P_{d k}} \hat{\boldsymbol{h}}_{k} \boldsymbol{u}_{k}, \sigma_{w_{k}}^{2}\right)$ where $\sigma_{w_{k}}^{2}=P_{d k} \sigma_{\tilde{\boldsymbol{h}}_{k}}^{2}+\sigma_{n}^{2}$. Therefore, we write $f\left(\boldsymbol{y}_{d k} \mid \boldsymbol{u}_{k}(i), \hat{\boldsymbol{h}}_{k}\right)$ as:

$$
f\left(\boldsymbol{y}_{d k} \mid \boldsymbol{u}_{k}(i), \hat{\boldsymbol{h}}_{k}\right)=\frac{1}{\sqrt{\pi \sigma_{w_{k}}^{2}}} \exp \left(-\frac{\left|\boldsymbol{y}_{d k}-\sqrt{P_{d k}} \hat{\boldsymbol{h}}_{k} e^{j 2 \pi \frac{i-1}{M}}\right|^{2}}{\sigma_{w_{k}}^{2}}\right)
$$

After eliminating the terms that are independent of $m$, the fusion rule reduces to $u_{0}=\arg \max _{m}$ $\pi_{m} \Theta_{m}$ where $\Theta_{m}$ is:

$$
\Theta_{m}=\prod_{k=1}^{N} \sum_{i=1}^{M} p_{i m} \exp \left(\frac{2 \sqrt{P_{d k}} R e\left(e^{\frac{-j 2 \pi(i-1)}{M}} \boldsymbol{y}_{d k} \hat{\boldsymbol{h}}_{k}^{*}\right)}{\sigma_{w_{k}}^{2}}\right)
$$


Note that the optimal fusion rule depends on $P_{k}, r_{d}$ (through $P_{d k}, \sigma_{w_{k}}^{2}$ ), channel outputs $\boldsymbol{y}_{d k}$, channel estimates $\hat{\boldsymbol{h}}_{k}$, and local sensor performance indices $p_{i m}$. For the special case of $M=2$, the optimal fusion rule reduces to:

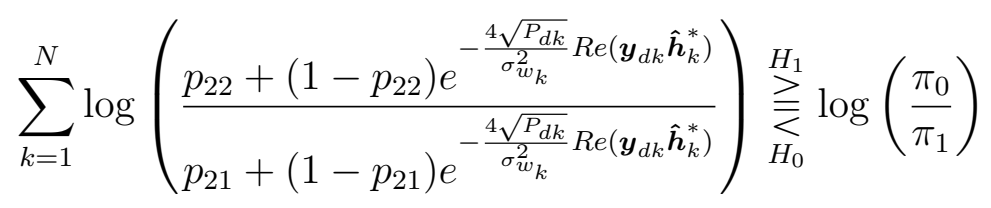

\section{Optimal Fusion Rule Corresponding to M-FSK}

To find $f\left(\underline{\boldsymbol{y}}_{d k} \mid \underline{\boldsymbol{u}}_{k}(i), \hat{\boldsymbol{h}}_{k}\right)$ in (2.6), we realize that given $\underline{\boldsymbol{u}}_{k}(i)$ and $\hat{\boldsymbol{h}}_{k}$, we have $\underline{\boldsymbol{y}}_{d k} \sim$ $C N\left(\sqrt{P_{d k}} \hat{\boldsymbol{h}}_{k} \underline{\boldsymbol{u}}_{k}(i), \boldsymbol{C}_{\underline{y}}\right)$ where $\boldsymbol{C}_{\underline{y}}$ is a diagonal matrix whose entries are $\boldsymbol{C}_{\underline{y}}(j, j)=\sigma_{n}^{2}$ for $j \neq i$ and $\boldsymbol{C}_{\underline{y}}(j, j)=\sigma_{w_{k}}^{2}$ for $j=i$. Therefore, we write $f\left(\underline{\boldsymbol{y}}_{d k} \mid \underline{\boldsymbol{u}}_{k}(i), \hat{\boldsymbol{h}}_{k}\right)$ as:

$$
\beta \exp \left(\sum_{j=1}^{M}-\frac{\left|\boldsymbol{y}_{d k}^{j}\right|^{2}}{\sigma_{n}^{2}}+\frac{\left|\boldsymbol{y}_{d k}^{i}\right|^{2}}{\sigma_{n}^{2}}-\frac{\left|\boldsymbol{y}_{d k}^{i}-\sqrt{P_{d k}} \hat{\boldsymbol{h}}_{k}\right|^{2}}{\sigma_{w_{k}}^{2}}\right)
$$

where $\boldsymbol{y}_{d k}^{i}$ denotes the $i$ th entry of vector $\underline{\boldsymbol{y}}_{d k}$ and $\beta^{-1}=\sqrt{\pi^{M} \operatorname{det}\left(\boldsymbol{C}_{\underline{y}}\right)}$. Eliminating the term $\exp \left(-\sum_{j=1}^{M} \frac{\left|\boldsymbol{y}_{d k}^{j}\right|^{2}}{\sigma_{n}^{2}}\right)$ inside the exp in (2.9), due to its irrelevance to $m$, the optimal fusion rule reduces to $u_{0}=\arg \max _{m} \pi_{m} \Theta_{m}$ where $\Theta_{m}$ is:

$$
\Theta_{m}=\prod_{k=1}^{N} \sum_{i=1}^{M} p_{i m} \underbrace{\exp \left(\frac{P_{d k} \sigma_{\tilde{\boldsymbol{h}}_{k}}^{2}\left|\boldsymbol{y}_{d k}^{i}\right|^{2}+2 \sqrt{P_{d k}} \sigma_{n}^{2} R e\left(\boldsymbol{y}_{d k}^{i} \hat{\boldsymbol{h}}_{k}^{*}\right)}{\sigma_{n}^{2} \sigma_{w_{k}}^{2}}\right)}_{=F\left(\boldsymbol{y}_{d k}^{i}\right)}
$$

Comparing (2.10) and (2.8) reveals the impact of the modulation scheme on the optimal fusion rule. For the special case of $M=2$ the optimal fusion rule reduces to:

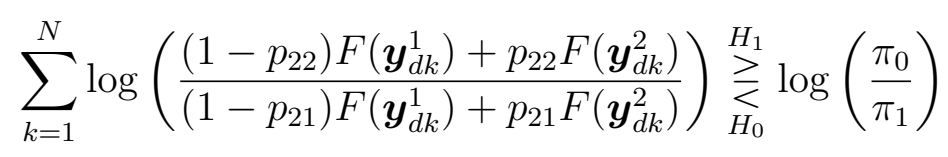




\section{Non Coherent Reception with $M$-FSK}

\section{Channel Amplitude Estimation}

The MMSE estimate of the channel amplitude $\boldsymbol{\alpha}_{k}$ given $\boldsymbol{v}_{t k}=\left|\boldsymbol{y}_{t k}^{1}\right|^{2}$ is $\hat{\boldsymbol{\alpha}}_{k}=E\left\{\boldsymbol{\alpha}_{k} \mid \boldsymbol{v}_{t k}\right\}=$ $\int \alpha_{k} f\left(\alpha_{k} \mid \boldsymbol{v}_{t k}\right) d \alpha_{k}$, where the conditional pdf $f\left(\boldsymbol{\alpha}_{k} \mid \boldsymbol{v}_{t k}\right)$ assumes the following form [20]:

$$
\begin{aligned}
f\left(\boldsymbol{\alpha}_{k} \mid \boldsymbol{v}_{t k}\right)= & 2 \boldsymbol{\alpha}_{k}\left(1+\gamma_{t k}\right) \exp \left(\frac{\gamma_{t k} \boldsymbol{v}_{t k}}{\left(1+\gamma_{t k}\right) \sigma_{n}^{2}}-\left(1+\gamma_{t k} \boldsymbol{\alpha}_{k}^{2}\right)\right) \\
& \times I_{0}\left(2 \boldsymbol{\alpha}_{k} \sqrt{\gamma_{t k} \frac{\boldsymbol{v}_{t k}}{\sigma_{n}^{2}}}\right)
\end{aligned}
$$

where $I_{0}($.$) is the modified Bessel functin of the first kind with order zero. Given \boldsymbol{v}_{t k}$, we have $\boldsymbol{\alpha}_{k} \sim \operatorname{Rice}\left(r, s^{2}\right)$ where $r=\frac{1}{\gamma_{t k}+1} \sqrt{\gamma_{t k} \frac{\boldsymbol{v}_{t k}}{\sigma_{n}^{2}}}$ and $s^{2}=\frac{1}{\gamma_{t k}+1}$. Therefore, $\hat{\boldsymbol{\alpha}}_{k}$ is [20]:

$$
\hat{\boldsymbol{\alpha}}_{k}=\frac{\sqrt{\pi s^{2}}}{2} F_{1}\left(\frac{-1}{2}, 1 ; \frac{-r^{2}}{s^{2}}\right)
$$

where $F_{1}(., . ;$.$) is the Kummer confluent hypergeometric function and F_{1}\left(\frac{-1}{2}, 1 ; x\right)=e^{\frac{x}{2}}\left(x I_{1}\left(\frac{x}{2}\right)-\right.$ $\left.(x-1) I_{0}\left(\frac{x}{2}\right)\right), I_{1}($.$) is the modified Bessel functin of the first kind with order one. Furthermore,$ the variance of estimation error can be computed as below [20]:

$$
\sigma_{\tilde{\alpha}_{k}}^{2}=1-\frac{\pi}{4} \frac{1}{\gamma_{t k}+1} E\left\{F_{1}\left(\frac{-1}{2}, 1 ; \frac{-r^{2}}{s^{2}}\right)^{2}\right\}
$$

Optimal Fusion Rule with Channel Amplitude Estimation

Substituting $\hat{\boldsymbol{\alpha}}_{k}$ in (2.3), we have:

$$
\underline{\boldsymbol{y}}_{d k}=\sqrt{P_{d k}} \hat{\boldsymbol{\alpha}}_{k} e^{j \phi_{k}} \underline{\boldsymbol{u}}_{k}+\underline{\boldsymbol{w}}_{k}, \text { where } \underline{\boldsymbol{w}}_{k}=\sqrt{P_{d k}} \tilde{\boldsymbol{\alpha}}_{k} e^{j \phi_{k}} \underline{\boldsymbol{u}}_{k}+\underline{\boldsymbol{n}}_{d k}
$$


To find $f\left(\underline{\boldsymbol{y}}_{d k} \mid \underline{\boldsymbol{u}}_{k}(i), \hat{\boldsymbol{\alpha}}_{k}\right)$ we write $f\left(\underline{\boldsymbol{y}}_{d k} \mid \underline{\boldsymbol{u}}_{k}(i), \hat{\boldsymbol{\alpha}}_{k}\right)=\int f\left(\underline{\boldsymbol{y}}_{d k} \mid \underline{\boldsymbol{u}}_{k}(i), \hat{\boldsymbol{\alpha}}_{k}, \phi\right) f(\phi) d \phi$. However, to express $f\left(\underline{\boldsymbol{y}}_{d k} \mid \underline{\boldsymbol{u}}_{k}(i), \hat{\boldsymbol{\alpha}}_{k}, \phi\right)$ we need the conditional pdf $f\left(\underline{\boldsymbol{w}}_{k} \mid \underline{\boldsymbol{u}}_{k}(i), \hat{\boldsymbol{\alpha}}_{k}, \phi\right)$. Unfortunately, this conditional pdf depends on the pdf $f\left(\hat{\boldsymbol{\alpha}}_{k}\right)$ and finding its closed form expression is mathematically intractable. However, our simulation results suggest that, conditional $\underline{\boldsymbol{w}}_{k}$ can be approximated as a zero-mean complex Gaussian vector with a diagonal covariance matrix $C_{\underline{w}}$ whose entries are $\boldsymbol{C}_{\underline{w}}(j, j)=\sigma_{n}^{2}$ for $j \neq i$ and $\boldsymbol{C}_{\underline{w}}(j, j)=\sigma_{w_{k}}^{2}=P_{d k} \sigma_{\tilde{\boldsymbol{\alpha}}_{k}}^{2}+\sigma_{n}^{2}$ for $j=i$. Consequently, given $\underline{\boldsymbol{u}}_{k}(i), \hat{\boldsymbol{\alpha}}_{k}$ and $\phi$, we can approximate $\underline{\boldsymbol{y}}_{d k} \sim C N\left(\sqrt{P_{d k}} \hat{\boldsymbol{\alpha}}_{k} e^{j \phi_{k}} \underline{\boldsymbol{u}}_{k}(i), \boldsymbol{C}_{\underline{w}}\right)$. With this approximation, we proceed with finding $f\left(\underline{\boldsymbol{y}}_{d k} \mid \underline{\boldsymbol{u}}_{k}(i), \hat{\boldsymbol{\alpha}}_{k}\right)$. One can verify the following:

$$
\begin{aligned}
& f\left(\underline{\boldsymbol{y}}_{d k} \underline{\boldsymbol{u}}_{k}(i), \hat{\boldsymbol{\alpha}}_{k}\right)=c_{1} c_{2}\left(\left|\boldsymbol{y}_{d k}^{i}\right|\right) \int_{0}^{2 \pi} \frac{1}{2 \pi} \exp \left(\frac{2 \sqrt{P_{d k}} \operatorname{Re}\left(\boldsymbol{y}_{d k}^{i} \hat{\boldsymbol{\alpha}}_{k} e^{-j \phi}\right)}{\sigma_{w_{k}}^{2}}\right) d \phi \\
& \stackrel{(a)}{=} \frac{c_{1} c_{2}\left(\left|\boldsymbol{y}_{d k}^{i}\right|\right)}{2 \pi} \int_{0}^{2 \pi} \exp \left(\frac{2 \sqrt{P_{d k}} \hat{\boldsymbol{\alpha}}_{k}\left|\boldsymbol{y}_{d k}^{i}\right| \cos (\phi-\theta)}{\sigma_{w_{k}}^{2}}\right) d \phi=c_{1} c_{2}\left(\left|\boldsymbol{y}_{d k}^{i}\right|\right) I_{0}\left(\frac{2 \sqrt{P_{d k}} \hat{\boldsymbol{\alpha}}_{k}}{\sigma_{w_{k}}^{2}}\left|\boldsymbol{y}_{d k}^{i}\right|\right)
\end{aligned}
$$

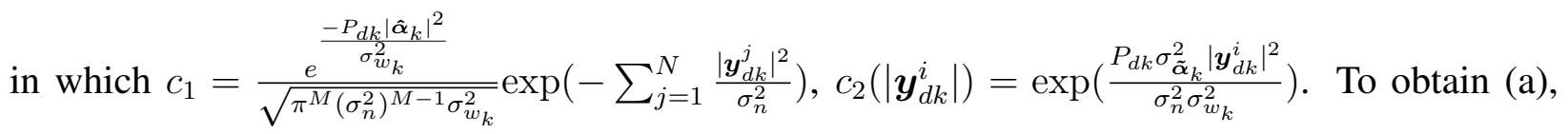
we let $\boldsymbol{y}_{d k}^{i}=\left|\boldsymbol{y}_{d k}^{i}\right| e^{j \theta}$. After substituting $f_{y}\left(\underline{\boldsymbol{y}}_{d k} \mid \underline{\boldsymbol{u}}_{k}(i), \hat{\boldsymbol{\alpha}}_{k}\right)$ in (2.6) and eliminating $c_{1}$ due to its irrelevance to $m$, the optimal fusion rule reduces to $u_{0}=\arg \max _{m} \pi_{m} \Theta_{m}$ where $\Theta_{m}$ is:

$$
\Theta_{m}=\prod_{k=1}^{N} \sum_{i=1}^{M} p_{i m} \underbrace{c_{2}\left(\left|\boldsymbol{y}_{d k}^{i}\right|\right) I_{0}\left(\frac{2 \sqrt{P_{d k}} \hat{\boldsymbol{\alpha}}_{k}}{\sigma_{w_{k}}^{2}}\left|\boldsymbol{y}_{d k}^{i}\right|\right)}_{=G\left(\left|\boldsymbol{y}_{d k}^{i}\right|\right)}
$$

Note that the optimal fusion rule depends on $P_{k}, r_{d}$ (through $P_{d k}, \sigma_{w_{k}}^{2}$ ), magnitude of channel outputs $\left|\boldsymbol{y}_{d k}^{i}\right|$, channel amplitude estimates $\hat{\boldsymbol{\alpha}}_{k}$, and local sensor performance indices $p_{i m}$. For the special case of $M=2$, the optimal fusion rule is similar to (2.11) with the difference that $F\left(\boldsymbol{y}_{d k}^{i}\right)$ needs to be replaced with $G\left(\left|\boldsymbol{y}_{d k}^{i}\right|\right)$ defined in (2.13). 


\section{Optimal Fusion Rule without Channel Amplitude Estimation}

In the absence of training, we have $P_{d}=P$ and $P_{t}=0$. To find $f\left(\underline{\boldsymbol{y}}_{d k} \mid \underline{\boldsymbol{u}}_{k}(i)\right)$ in (2.6), we realize that given $\underline{\boldsymbol{u}}_{k}(i)$, we have $\underline{\boldsymbol{y}}_{d k} \sim C N\left(0, \boldsymbol{C}_{\underline{y}}\right)$ where $\boldsymbol{C}_{\underline{y}}$ is a diagonal matrix whose entries are $\boldsymbol{C}_{\underline{y}}(j, j)=\sigma_{n}^{2}$ for $j \neq i$ and $\boldsymbol{C}_{\underline{y}}(j, j)=P_{d k} \sigma_{h}^{2}+\sigma_{n}^{2}$ for $j=i$. We can verify that $f\left(\underline{\boldsymbol{y}}_{d k} \mid \underline{\boldsymbol{u}}_{k}(i)\right)$ equals to

$$
\beta \exp \left(\frac{P_{d k} \sigma_{h}^{2}\left|\boldsymbol{y}_{d k}^{i}\right|^{2}}{\sigma_{n}^{2}\left(\sigma_{n}^{2}+P_{d k} \sigma_{h}^{2}\right)}\right) \prod_{j=1}^{M} \exp \left(-\frac{\left|\boldsymbol{y}_{d k}^{j}\right|^{2}}{\sigma_{n}^{2}}\right)
$$

After substituting $f_{y}\left(\underline{\boldsymbol{y}}_{d k} \mid \underline{\boldsymbol{u}}_{k}(i)\right)$ in (2.6) and eliminating $\prod_{j=1}^{M} \exp \left(-\frac{\left|\boldsymbol{y}_{d k}^{j}\right|^{2}}{\sigma_{n}^{2}}\right)$ due to its irrelevance to $m$, the optimal fusion rule reduces to $u_{0}=\arg \max _{m}$ $\pi_{m} \Theta_{m}$ where $\Theta_{m}$ is:

$$
\Theta_{m}=\prod_{k=1}^{N} \sum_{i=1}^{M} p_{i m} \underbrace{\exp \left(\frac{P_{d k} \sigma_{h}^{2}\left|\boldsymbol{y}_{d k}^{i}\right|^{2}}{\sigma_{n}^{2}\left(\sigma_{n}^{2}+P_{d k} \sigma_{h}^{2}\right)}\right)}_{=H\left(\left|\boldsymbol{y}_{d k}^{i}\right|\right)}
$$

Different from (2.13), (2.14) does not depend on channel amplitude estimates and only depends on the channel statistics. For the special case of $M=2$, the optimal fusion rule is similar to (2.11) with the difference that $F\left(\boldsymbol{y}_{d k}^{i}\right)$ needs to be replaced with $H\left(\left|\boldsymbol{y}_{d k}^{i}\right|\right)$ defined in (2.14). 


\section{CHAPTER 3: OPTIMAL FUSION RULE FOR M-ARY DISTRIBUTED DETECTION WITH BINARY MODULATION}

In this chapter, we consider an $M$-ary distributed detection system, in which sensors send their decisions over orthogonal noisy channels with Rayleigh and Rician fading channel model to a fusion center. In chapter 2, we used M-PSK or M-FSK modulation for sending data from sensors to FC. In this chapter we use BPSK or BFSK for sending data to FC. We derive the optimal fusion rule for coherent reception and investigate the effect of imperfect channel state information on the fusion rules and detection performance, assuming the sum of training and data symbol transmit powers is fixed. This chapter is organized as follows. First, we present our system model. Next, for Rayleigh and Rician fading channel models, we derive the optimal fusion rules.

\section{System Model and Problem Statement}

We consider the problem of testing which of the $M \geq 2$ hypotheses $\left\{H_{j}\right\}_{j=1}^{M}$ has been occurred. The sensing model is similar to sensing model in chapter 2. Each sensor makes a decision and forwards its decision to FC. Sensor $k$ employs BPSK or BFSK modulation to map its $M$-ary decision to an $L$-bit sequence $\underline{\boldsymbol{u}}_{k}=\left[\boldsymbol{u}_{k}(1), \ldots, \boldsymbol{u}_{k}(L)\right]$ for BPSK and $\underline{\boldsymbol{u}}_{k}=\left[\underline{\boldsymbol{u}}_{k}(1), \ldots, \underline{\boldsymbol{u}}_{k}(L)\right]$ for BFSK modulation where $\boldsymbol{u}_{k}(l) \in\{-1,1\}$ for BPSK and $\underline{\boldsymbol{u}}_{k}(l) \in\left\{\left[\begin{array}{ll}1 & 0\end{array}\right],\left[\begin{array}{ll}0 & 1\end{array}\right]\right\}$ for BFSK modulation. Each sensor transmits this binary sequence with power $P_{d}$. Assuming $\underline{\boldsymbol{u}}_{k}$ 's are sent over orthogonal channels between sensors and the FC, the channel output corresponding to sensor $k$ at the FC upon the reception of $\underline{\boldsymbol{u}}_{k}$ is $\underline{\boldsymbol{y}}_{d k}=\left[\boldsymbol{y}_{d k}(1), \ldots, \boldsymbol{y}_{d k}(L)\right]$ for BPSK and $\underline{\boldsymbol{y}}_{d k}=\left[\underline{\boldsymbol{y}}_{d k}(1), \ldots, \underline{\boldsymbol{y}}_{d k}(L)\right]$ 
for BFSK where $\boldsymbol{y}_{d k}(l)$ and $\underline{\boldsymbol{y}}_{d k}(l)$ are

$$
\begin{gathered}
\boldsymbol{y}_{d k}(l)=\sqrt{P_{d k}} \boldsymbol{h}_{k} \boldsymbol{u}_{k}(l)+\boldsymbol{n}_{d k}(l) \quad \text { BPSK, } \\
\underline{\boldsymbol{y}}_{d k}(l)=\sqrt{P_{d k}} \boldsymbol{h}_{k} \underline{\boldsymbol{u}}_{k}(l)+\underline{\boldsymbol{n}}_{d k}(l) \quad \mathrm{BFSK}, \\
l \in\{1, \ldots, L\}, L=\log _{2} M
\end{gathered}
$$

where $P_{d k}=G P_{d}\left(\Delta_{k}\right)^{-\epsilon}$ is the received power corresponding to the data symbol, $G$ is a constant, $\epsilon$ is the path loss exponent, and $\Delta_{k}$ is the distance between sensor $k$ and the FC. The communication channel noises, denoted as $\boldsymbol{n}_{d k}(l)$ and $\underline{\boldsymbol{n}}_{d k}(l)$, are zero mean complex Gaussian $\boldsymbol{n}_{d k} \sim C N\left(0, \sigma_{n}^{2}\right)$, $\underline{\boldsymbol{n}}_{d k} \sim C N\left(0, \sigma_{n}^{2} I\right)$, where $I$ is a $2 \times 2$ identity matrix. We assume that the channel outputs conditioned on the channel inputs, are independent across the sensors. We represent the complex channel coefficient in (3.1) as $\boldsymbol{h}_{k}=\boldsymbol{\alpha}_{k} e^{j \phi_{k}}$ where $\alpha_{k}$ and $\phi_{k}$, respectively, are the channel amplitude and phase. We assume $\boldsymbol{h}_{k} \sim C N\left(\bar{\mu}_{k}, \sigma_{h}^{2}\right)$ where $\bar{\mu}_{k}$ is the Rice factor, $\bar{\mu}_{k}=A_{k} e^{j \theta_{k}}$ with $A_{k}$ and $\theta_{k}$ being the amplitude and phase shift of the line of sight (LOS) component. This model encompasses Rician and Rayleigh fading with $E\left\{\left|\boldsymbol{h}_{k}\right|^{2}\right\}=\sigma_{h}^{2}$. For Rayleigh fading (i.e., there is no LOS component), the channel distribution reduces to $\boldsymbol{h}_{k} \sim C N\left(0, \sigma_{h}^{2}\right)$.

To enable training based channel estimation, we assume that the channel coefficients are fixed during sending $L$ binary data symbols, and each sensor sends a training symbol with power $P_{t}$ along with its $L$ data symbols. We refer to the symbols $u_{t}, \underline{u}_{t}$ as training symbols and $P_{t}$ as training symbol transmit power. Without loss of generality, we assume $u_{t}=1$ and $\underline{u}_{t}=\underline{e}_{1}$, respectively, when the sensors employ BPSK and BFSK schemes. Training symbols are also sent over orthogonal channels between sensors and the FC, prior to sending data symbols. The channel output 
corresponding to sensor $k$ at the FC upon the reception of training symbol is:

$$
\begin{aligned}
& \boldsymbol{y}_{t k}=\sqrt{P_{t k}} \boldsymbol{h}_{k} u_{t}+\boldsymbol{n}_{t k} \quad \mathrm{BPSK}, \\
& \underline{\boldsymbol{y}}_{t k}=\sqrt{P_{t k}} \boldsymbol{h}_{k} \underline{u}_{t}+\underline{\boldsymbol{n}}_{t k} \quad \mathrm{BFSK},
\end{aligned}
$$

where the noises $\boldsymbol{n}_{t k} \sim C N\left(0, \sigma_{n}^{2}\right), \underline{\boldsymbol{n}}_{t k} \sim C N\left(0, \sigma_{n}^{2} I\right)$ are independent from $\boldsymbol{n}_{d k}$ and $\underline{\boldsymbol{n}}_{d k}$ in (3.1). Also, $P_{t k}=G P_{t}\left(\Delta_{k}\right)^{-\epsilon}$ is the received power corresponding to the training symbol. We consider coherent receiver with a training based channel estimator where the unknown parameter to be estimated is $\boldsymbol{h}_{k}$. We model $\boldsymbol{h}_{k}$ as $\boldsymbol{h}_{k}=\hat{\boldsymbol{h}}_{k}+\tilde{\boldsymbol{h}}_{k}$, where $\hat{\boldsymbol{h}}_{k}$ is the channel estimate based on $\boldsymbol{y}_{t k}$ and $\underline{\boldsymbol{y}}_{t k}$ in (3.2), and $\tilde{\boldsymbol{h}}_{k}$ is the estimation error ${ }^{1}$. To include the cost of channel estimation, we assume that the transmit power consumption per decision $P_{t}+P_{d}=P$ is constant. We define $P_{k}=G P\left(\Delta_{k}\right)^{-\epsilon}$ and let $r_{d}=P_{d} / P=P_{d k} /\left(P_{d k}+P_{t k}\right)=P_{d k} / P_{k}$, where $r_{d} \in[0,1]$ is the fraction of the power assigned to the data symbol.

We adopt the Bayesian criterion to find the optimal fusion rule at the FC, in order to make a global decision $u_{0} \in\left\{H_{1}, H_{2}, . ., H_{M}\right\}$. The optimal fusion rule is $u_{0}=\arg \max _{m} \pi_{m} \Theta_{m}$ where $\Theta_{m}$ varies, depending on the modulation scheme. Since the channel outputs are independent across sensors, we find:

$$
\Theta_{m}=\prod_{k=1}^{N} \sum_{i=1}^{M} \prod_{l=1}^{L} p_{i m} f\left(\boldsymbol{y}_{d k}(l) \mid \boldsymbol{u}_{k}^{i}(l), \hat{\boldsymbol{h}}_{k}\right)
$$

when sensors employ BPSK. When sensors employ BFSK we obtain:

$$
\Theta_{m}=\prod_{k=1}^{N} \sum_{i=1}^{M} \prod_{l=1}^{L} p_{i m} f\left(\underline{\boldsymbol{y}}_{d k} \mid \underline{\boldsymbol{u}}_{k}^{i}(l), \hat{\boldsymbol{h}}_{k}\right)
$$

\footnotetext{
${ }^{1}$ Since $\underline{u}_{t}=\underline{e}_{1}$, the estimator only employs the first entry of vector $\underline{\boldsymbol{y}}_{t k}$, denoted as $\boldsymbol{y}_{t k}^{1}$, for channel amplitude estimation.
} 
where $\boldsymbol{u}_{k}^{i}(l), \underline{\boldsymbol{u}}_{k}^{i}(l)$ in (3.3), (3.4) are the transmitted $l$ th binary data symbols of $\underline{\boldsymbol{u}}_{k}$ for sensor $k$ corresponding to the decision of $H_{i}$ and $p_{i m}$ is obtained from (2.2). Since the sum of transmit powers of training and data symbols is fixed, the estimation error and thus the performance of the optimal fusion rules would depend on $P_{k}$ and the ratio $r_{d}$.

\section{Coherent Reception with BPSK and BFSK}

\section{Complex Channel Estimation}

For the linear signal model in (3.2), the MMSE channel estimation of $\boldsymbol{h}_{k}$ given $\boldsymbol{y}_{t k}$ is $\hat{\boldsymbol{h}}_{k}=$ $E\left\{\boldsymbol{h}_{k} \mid \boldsymbol{y}_{t k}\right\}=\bar{\mu}_{k}+\frac{\sigma_{h}^{2} \sqrt{P_{t k}}}{\sigma_{h}^{2} P_{t k}+\sigma_{n}^{2}}\left(\boldsymbol{y}_{t k}-\sqrt{P_{t k}} \bar{\mu}_{k}\right)$. Substituting $\hat{\boldsymbol{h}}_{k}$ in $\boldsymbol{y}_{d k}$ in (3.1), we find:

$$
\boldsymbol{y}_{d k}=\sqrt{P_{d k}} \hat{\boldsymbol{h}}_{k} \boldsymbol{u}_{k}+\boldsymbol{w}_{k}, \text { where } \boldsymbol{w}_{k}=\sqrt{P_{d k}} \tilde{\boldsymbol{h}}_{k} \boldsymbol{u}_{k}+\boldsymbol{n}_{d k}
$$

The complex noise $\boldsymbol{w}_{k}$ in (3.5) combines the AWGN noise and the channel estimation error. Considering (3.2), we observe that $\boldsymbol{y}_{t k} \sim C N\left(0, P_{t k} \sigma_{h}^{2}+\sigma_{n}^{2}\right)$. Let $\gamma_{t k}=P_{t k} / \sigma_{n}^{2}$. Since $\hat{\boldsymbol{h}}_{k}$ is a linear function of $\boldsymbol{y}_{t k}$, we have $\hat{\boldsymbol{h}}_{k} \sim C N\left(\bar{\mu}_{k}, \frac{\sigma_{h}^{4} \gamma_{t k}}{1+\sigma_{h}^{2} \gamma_{t k}}\right)$ and $\tilde{\boldsymbol{h}}_{k} \sim C N\left(0, \frac{\sigma_{h}^{2}}{1+\sigma_{h}^{2} \gamma_{t k}}\right)$ [20].

\section{Optimal Fusion Rule Corresponding to BPSK}

To find $f\left(\boldsymbol{y}_{d k}(l) \mid \boldsymbol{u}_{k}^{i}(l), \hat{\boldsymbol{h}}_{k}\right)$ in (3.3), we realize that given $\boldsymbol{u}_{k}(l)$ and $\hat{\boldsymbol{h}}_{k}$, we have $\boldsymbol{y}_{d k}(l) \sim C N$ $\left(\sqrt{P_{d k}} \hat{\boldsymbol{h}}_{k} \boldsymbol{u}_{k}(l), \sigma_{w_{k}}^{2}\right)$ where $\sigma_{w_{k}}^{2}=P_{d k} \sigma_{\tilde{h}_{k}}^{2}+\sigma_{n}^{2}$. Therefore, we write $f\left(\boldsymbol{y}_{d k}(l) \mid \boldsymbol{u}_{k}^{i}(l), \hat{\boldsymbol{h}}_{k}\right)$ as:

$$
f\left(\boldsymbol{y}_{d k}(l) \mid \boldsymbol{u}_{k}^{i}(l), \hat{\boldsymbol{h}}_{k}\right)=\frac{1}{\sqrt{\pi \sigma_{w_{k}}^{2}}} \exp \left(-\frac{\left|\boldsymbol{y}_{d k}(l)-\sqrt{P_{d k}} \hat{\boldsymbol{h}}_{k} \boldsymbol{u}_{k}^{i}(l)\right|^{2}}{\sigma_{w_{k}}^{2}}\right)
$$


After eliminating the terms that are independent of $m$, the fusion rule reduces to $u_{0}=\arg \max _{m}$ $\pi_{m} \Theta_{m}$ where $\Theta_{m}$ is:

$$
\Theta_{m}=\prod_{k=1}^{N} \sum_{i=1}^{M} P_{i m}^{k} \exp \left(\frac{2 \sqrt{P_{d k}} R e\left(\sum_{l=1}^{L} \hat{h}_{k}^{*} \boldsymbol{y}_{d k}(l) \boldsymbol{u}_{k}^{i}(l)\right)}{\sigma_{w_{k}}^{2}}\right)
$$

Note that the optimal fusion rule depends on $P_{k}, r_{d}$ (through $P_{d k}, \sigma_{w_{k}}^{2}$ ), channel outputs $\boldsymbol{y}_{d k}(l)$, channel estimates $\hat{\boldsymbol{h}}_{k}$, and local sensor performance indices $p_{i m}$.

Low SNR approximation: As the channel noise variance $\sigma_{n}^{2} \rightarrow \infty$, we have $\sigma_{\tilde{\boldsymbol{h}}_{k}}^{2} \rightarrow \sigma_{h}^{2}$ and $\sigma_{w_{k}}^{2} \rightarrow \infty$. Using the approximations $e^{-x} \approx 1-x$ for small $x$ and the fact that $\sum_{i=1}^{M} p_{i m}=1$, we can simplify $\Theta_{m}$ in (3.6) as the following:

$$
\Theta_{m}=\prod_{k=1}^{N} \sum_{i=1}^{M} p_{i m}\left(\frac{2 \sqrt{P_{d k}} R e\left(\sum_{l=1}^{L} \hat{h}_{k}^{*} \boldsymbol{y}_{d k}(l) \boldsymbol{u}_{k}^{i}(l)\right)}{\sigma_{w_{k}}^{2}}\right)
$$

Special case of $M=2$ : The optimal fusion rule reduces to:

$$
\sum_{k=1}^{N} \log \left(\frac{p_{22}+\left(1-p_{22}\right) e^{-\frac{4 \sqrt{P_{d k}}}{\sigma_{w_{k}}^{2}}} R e\left(\boldsymbol{y}_{d k} \hat{\boldsymbol{h}}_{k}^{*}\right)}{p_{21}+\left(1-p_{21}\right) e^{-\frac{4 \sqrt{P_{d k}}}{\sigma_{w_{k}}^{2}}} \operatorname{Re}\left(\boldsymbol{y}_{d k} \hat{\boldsymbol{h}}_{k}^{*}\right)}\right) \underset{H_{0}}{\gtrless} \log \left(\frac{\pi_{0}}{\pi_{1}}\right)
$$

\section{Optimal Fusion Rule Corresponding to BFSK}

To find $f\left(\underline{\boldsymbol{y}}_{d k} \mid \underline{\boldsymbol{u}}_{k}^{i}(l), \hat{\boldsymbol{h}}_{k}\right)$ in (3.4), we realize that given $\underline{\boldsymbol{u}}_{k}(l)$ and $\hat{\boldsymbol{h}}_{k}$, we have $\underline{\boldsymbol{y}}_{d k}(l) \sim C N$ $\left(\sqrt{P_{d k}} \hat{\boldsymbol{h}}_{k} \underline{\boldsymbol{u}}_{k}^{i}(l), \boldsymbol{C}_{\underline{y}}\right)$ where $\boldsymbol{C}_{\underline{y}}$ is a $2 \times 2$ diagonal matrix whose entries are $\boldsymbol{C}_{\underline{y}}(j, j)=\sigma_{n}^{2}$ for $j \neq i$ and $\boldsymbol{C}_{\underline{y}}(j, j)=\sigma_{w_{k}}^{2}$ for $j=i$. Therefore, we write $f\left(\underline{\boldsymbol{y}}_{d k}(l) \mid \underline{\boldsymbol{u}}_{k}^{i}(l), \hat{\boldsymbol{h}}_{k}\right)$ as

$$
\beta \exp \left(\sum_{j=1}^{2}-\frac{\left|\boldsymbol{y}_{d k}^{j}(l)\right|^{2}}{\sigma_{n}^{2}}+\frac{\left|\boldsymbol{y}_{d k}^{i}(l)\right|^{2}}{\sigma_{n}^{2}}-\frac{\left|\boldsymbol{y}_{d k}^{i}-\sqrt{P_{d k}} \hat{\boldsymbol{h}}_{k}\right|^{2}}{\sigma_{w_{k}}^{2}}\right)
$$


where $\boldsymbol{y}_{d k}^{i}(l)$ denotes the $i$ th entry of vector $\underline{\boldsymbol{y}}_{d k}(l)$ and $\beta^{-1}=\sqrt{\pi^{M} \operatorname{det}\left(\boldsymbol{C}_{\underline{y}}\right)}$. Eliminating the term $\exp \left(-\sum_{j=1}^{2} \frac{\left|\boldsymbol{y}_{d k}^{j}(l)\right|^{2}}{\sigma_{n}^{2}}\right)$ inside the exp in (3.7), due to its irrelevance to $m$, the optimal fusion rule reduces to $u_{0}=\arg \max _{m} \pi_{m} \Theta_{m}$ where $\Theta_{m}$ is

$$
\Theta_{m}=\prod_{k=1}^{N} \sum_{i=1}^{M} p_{i m} \exp \left(\frac{\sum_{l=1}^{L} P_{d k} \sigma_{\tilde{h}_{k}}^{2}\left\|\underline{\boldsymbol{y}}_{d k}(l) \cdot \underline{\boldsymbol{u}}_{k}^{i}(l)\right\|^{2}}{\sigma_{n}^{2} \sigma_{w_{k}}^{2}}+\frac{\sum_{l=1}^{L} 2 \sqrt{P_{d k}} R e\left(\hat{h}_{k}^{*} \underline{\boldsymbol{y}}_{d k}(l) \cdot \underline{\boldsymbol{u}}_{k}^{i}(l)\right)}{\sigma_{w_{k}}^{2}}\right)
$$

Comparing (3.8) and (3.6) reveals the impact of the modulation scheme on the optimal fusion rule. Low SNR approximation: Using similar low SNR approximations for BPSK, we can simplify $\Theta_{m}$ in (3.8) as below:

$$
\Theta_{m}=\prod_{k=1}^{N} \sum_{i=1}^{M} p_{i m}\left(\frac{\sum_{l=1}^{L} P_{d k} \sigma_{\tilde{h}_{k}}^{2}\left\|\underline{\boldsymbol{y}}_{d k}(l) \cdot \underline{\boldsymbol{u}}_{k}^{i}(l)\right\|^{2}}{\sigma_{n}^{2} \sigma_{w_{k}}^{2}}+\frac{\sum_{l=1}^{L} \sqrt{2} P_{d k} R e\left(\hat{h}_{k}^{*} \underline{\boldsymbol{y}}_{d k}(l) \cdot \underline{\boldsymbol{u}}_{k}^{i}(l)\right)}{\sigma_{w_{k}}^{2}}\right)
$$

Special case of $M=2$ : The optimal fusion rule reduces to:

$$
\sum_{k=1}^{N} \log \left(\frac{\left(1-p_{22}^{k}\right) F\left(\boldsymbol{y}_{d k}(1)\right)+p_{22}^{k} F\left(\boldsymbol{y}_{d k}(2)\right)}{\left(1-p_{21}^{k}\right) F\left(\boldsymbol{y}_{d k}(1)\right)+p_{21}^{k} F\left(\boldsymbol{y}_{d k}(2)\right)}\right) \underset{H_{0}}{\gtrless} \log \left(\frac{\pi_{0}}{\pi_{1}}\right)
$$

where $F(x)=\frac{P_{d k} \sigma_{\tilde{h}_{k}}^{2}\|x\|^{2}+2 \sigma_{n}^{2} \sqrt{P_{d k}} R e\left(\hat{h}_{k}^{*} x\right)}{2 \sigma_{n}^{2} \sigma_{w_{k}}^{2}}$. 


\section{CHAPTER 4: NUMERICAL RESULTS}

To conduct our numerical simulations, we let $M=4$ and assume $\pi_{1}=\pi_{2}=\pi_{3}=\pi_{4}=1 / 4$. For the means $m_{j}$ of Gaussian sources we assume $m_{j} \in\{-2 v,-v, v, 2 v\}$ where $v=5 \times 10^{-5}$. We let $N=5, G=-30 \mathrm{~dB}, \epsilon=2, \sigma_{n}^{2}=-50 \mathrm{~dB}$. We assume all sensors are equally distant from the FC with $\Delta_{k}=10 m$ and define $P_{t k}=\bar{P}_{t}, P_{d k}=\bar{P}_{d}, P_{k}=\bar{P}$ for all $k$ and $S N R=10 \log \left(\frac{\bar{P}}{\sigma_{n}^{2}}\right)$.

Figures (4.1) and (4.2) represent error probability $\left(P_{e}\right)$ when sensors employ $M$-ary modualtion for sending their data to FC. Figures 4.1(a) and 4.2(a) depict error probability vs $S N R$ for coherent and non coherent reception, respectively. As a benchmark, we also plot $P_{e}$ assuming perfect CSI at the FC. The gap between perfect CSI and imperfect CSI at low $S N R$ in coherent reception is noticeable, which is due to the channel estimation error. However, as $S N R$ increases the channel estimation error reduces and this gap goes to zero. For non coherent reception, we observe that the lack of perfect knowledge of channel amplitude increases $P_{e}$. Furthermore, in the case of coherent reception, we observe that $P_{e}$ is lower in FSK, compared to PSK since 4-FSK represents the signal in more dimensions, compared to 4-PSK. Figures 4.1(b) and 4.2(b) show $P_{e}$ vs $r_{d}$ for coherent and non coherent reception, respectively. We observe that for FSK modulation (regardless of the reception mode at the FC) as $r_{d}$ increases, $P_{e}$ decreases. In fact, the optimal $r_{d}$ is one, implying that no transmit power should be allocated for sending training symbol. On the other hand, for PSK modulation and coherent reception $P_{e}$ is minimized at $r_{d}=0.5$. Figures (4.3) and (4.4) represent $P_{e}$ at the FC when sensors employ binary modulation for sending their data. Figures 4.3(a) and 4.4(a) show that the performance gap between perfect CSI and imperfect CSI at low $S N R$ for both channel models is noticeable. However, as $S N R$ increases the channel estimation error reduces and this gap goes to zero. Also, BPSK appears to be more vulnerable to channel estimation error, as the performance gap between perfect CSI and imperfect CSI for BPSK is larger than that of BFSK. Furthermore, we observe that $P_{e}$ corresponding to BPSK is lower than that of BFSK. 
Figures 4.3(b) and 4.4(b) show $P_{e}$ vs $r_{d}$ for Rayleigh and Rician fading channel models, respectively. We observe that for Rician fading model (regardless of modulation) and for Rayleigh fading model with BFSK modulation, as $r_{d}$ increases $P_{e}$ decreases. In fact, the optimal $r_{d}$ is one, implying that each sensor should allocate all its transmit power to its $L$ data symbols. On the other hand, for Rayleigh fading model with BPSK modulation $P_{e}$ is minimized at $r_{d}=0.5$, i.e., each sensor should allot half of its transmit power for training symbol. The reason for these different behaviors is the different levels of vulnerability of these two modulations to signal distortion due to the random channel phase. For PSK modulation, a phase shift (due to random channel phase) decreases the Euclidean distance between the two constellation points and hence $P_{e}$ increases. Whereas, in FSK modulation a phase shift does not change this distance. In fact, low data power (small $\left.r_{d}\right)$ can be much more detrimental than low training power (large $r_{d}$ ) for FSK modulation for both coherent and non coherent reception. 


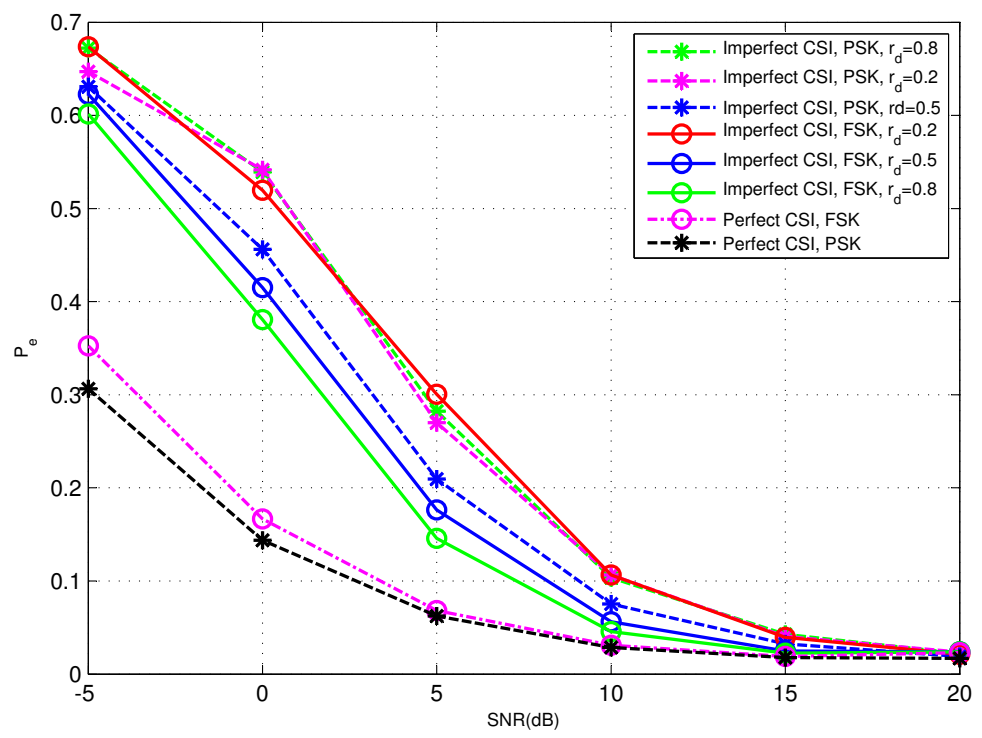

(a)

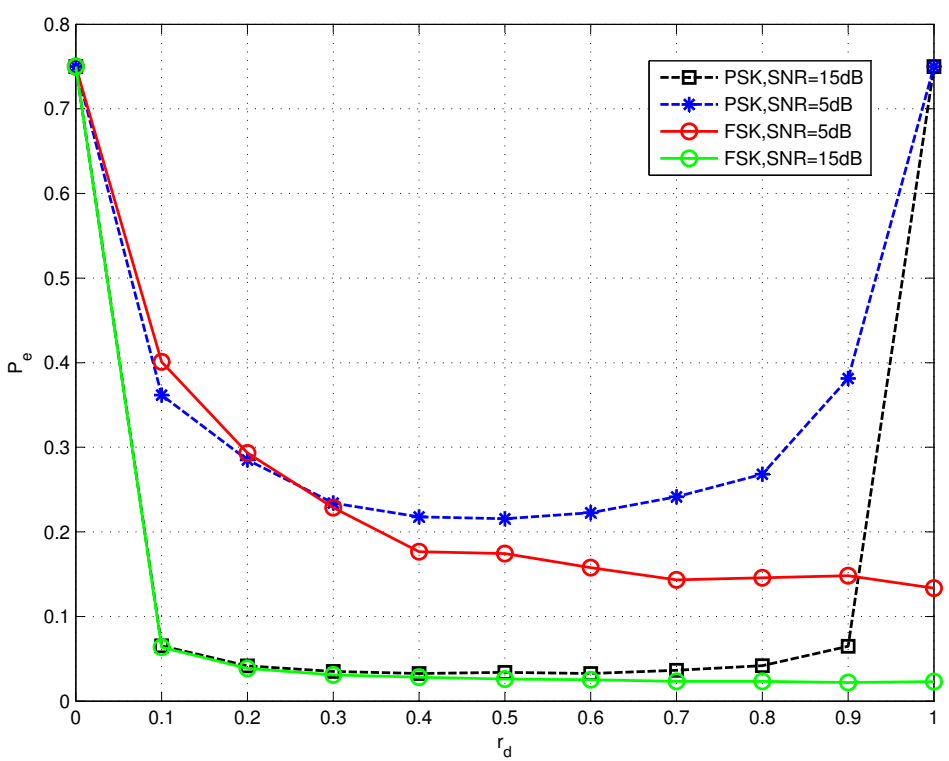

(b)

Figure 4.1: Training based coherent reception with 4-PSK and 4-FSK modulation: (a) $P_{e}$ vs. SNR, (b) $P_{e}$ vs. $r_{d}$. 


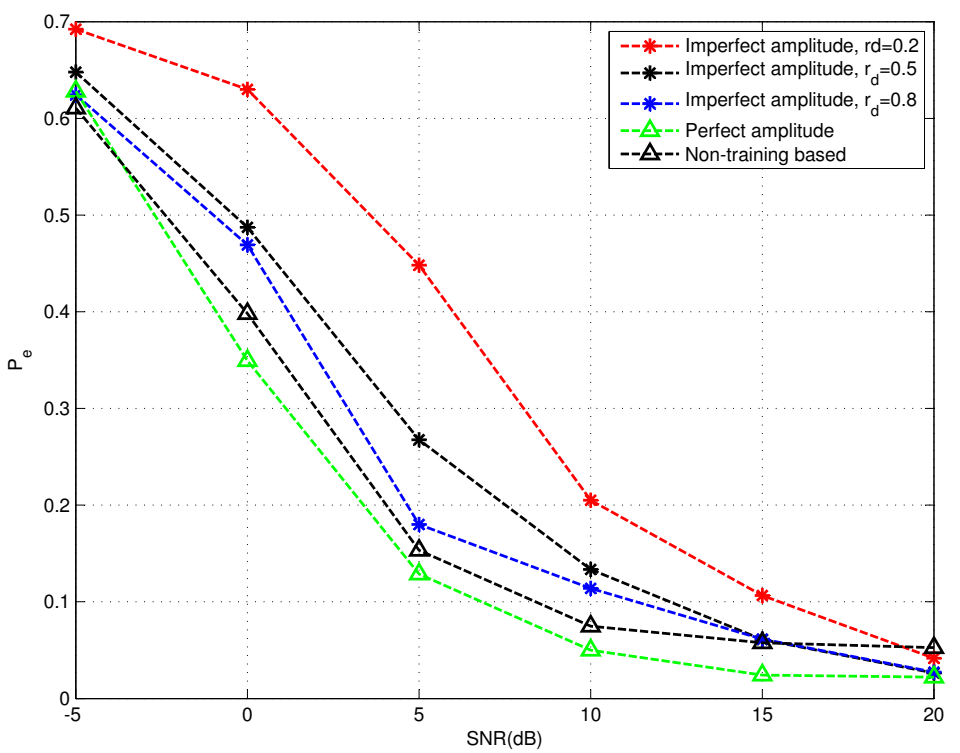

(a)

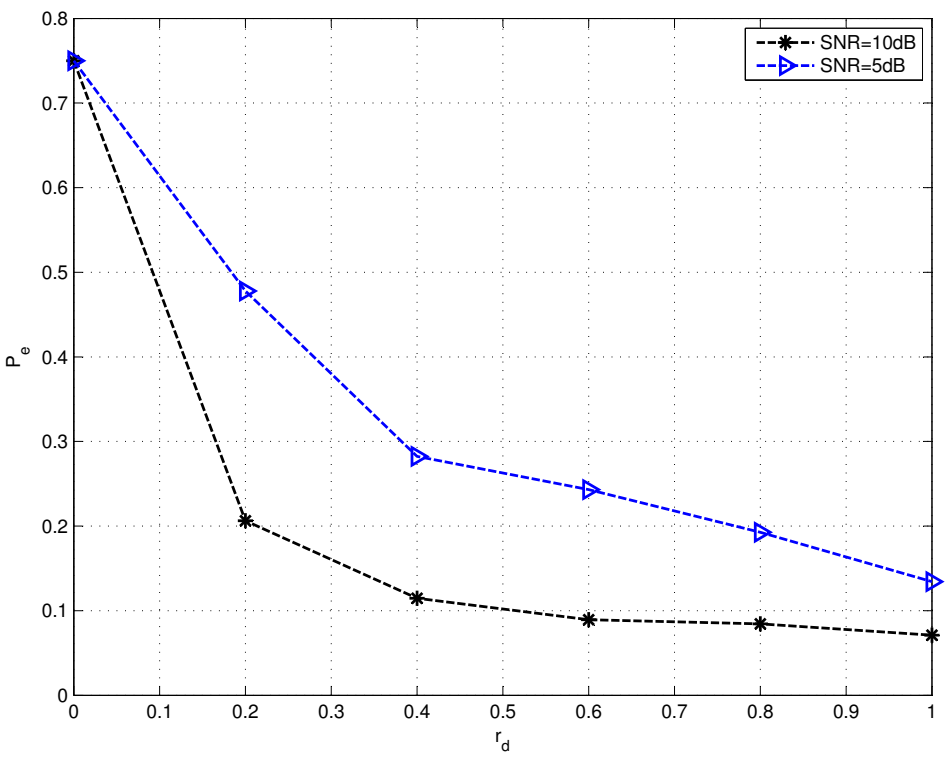

(b)

Figure 4.2: Noncoherent reception with 4-FSK modulation: (a) $P_{e}$ vs. SNR for training and nontraining based, (b) $P_{e}$ vs. $r_{d}$ for training based. 


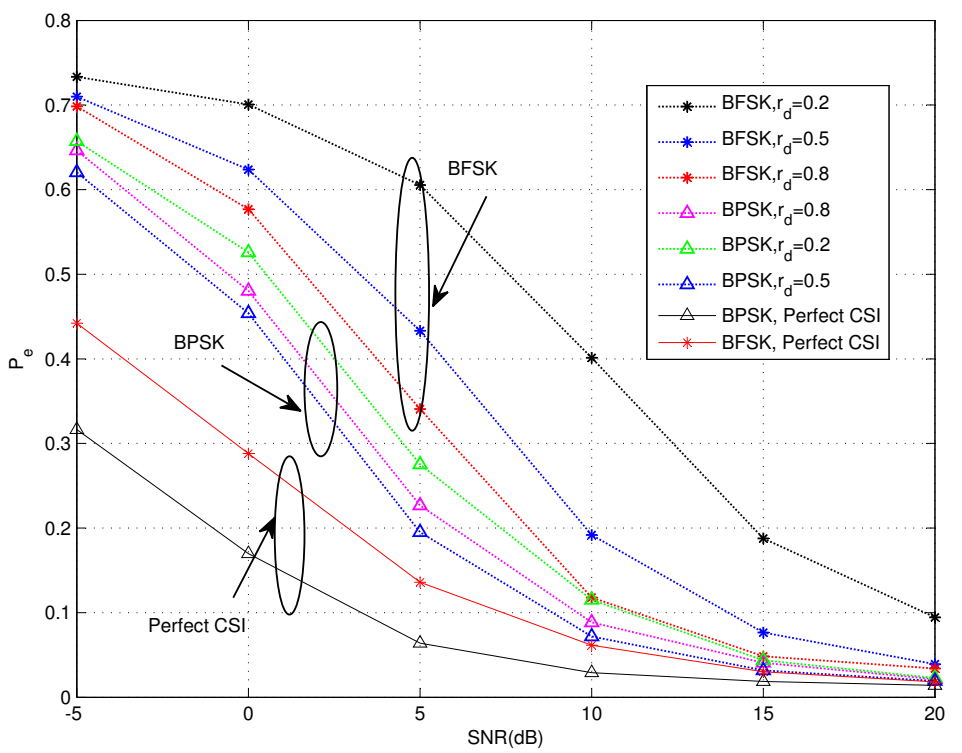

(a)

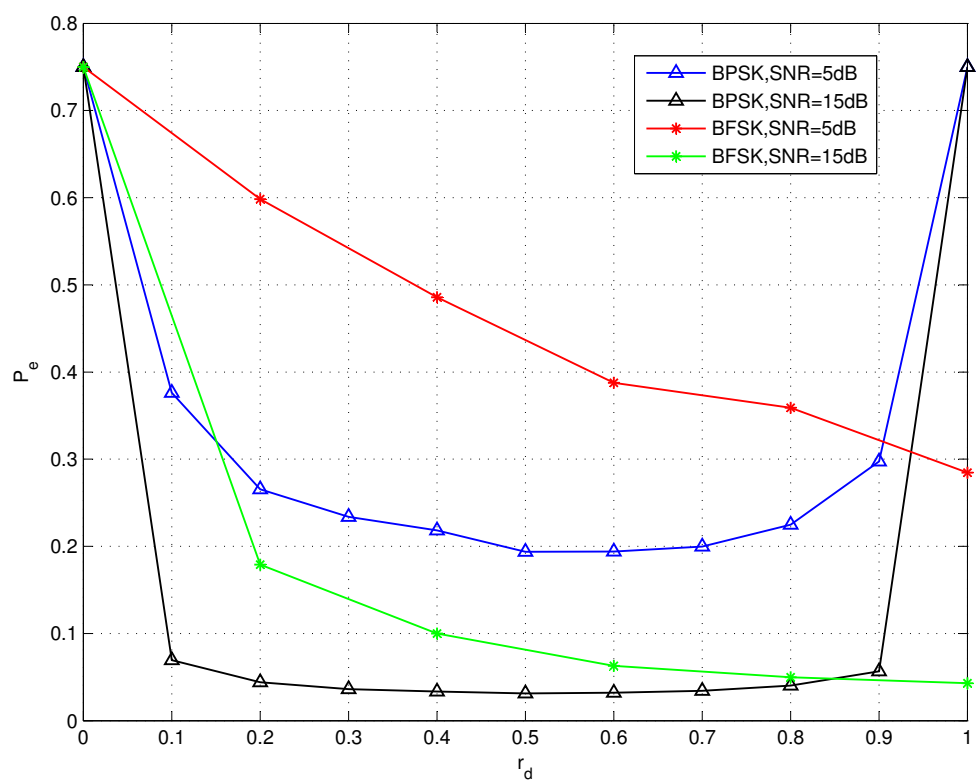

(b)

Figure 4.3: Rayleigh fading channel model with BPSK and BFSK modulation: (a) $P_{e}$ vs. SNR, (b) $P_{e}$ vs. $r_{d}$. 


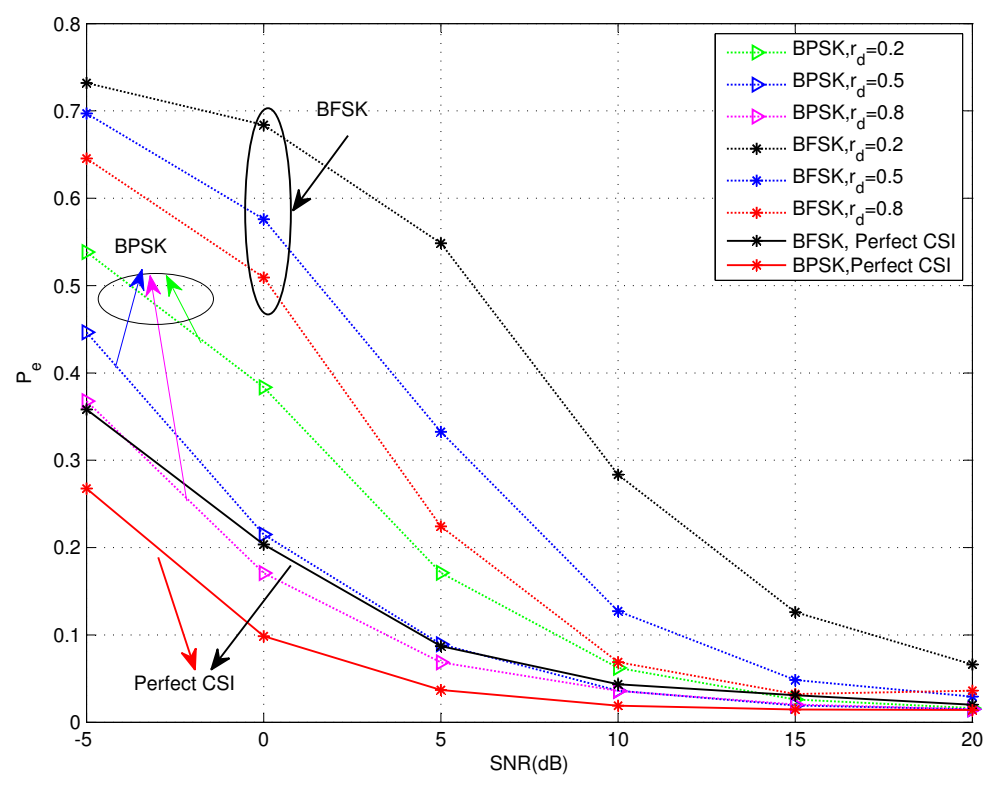

(a)

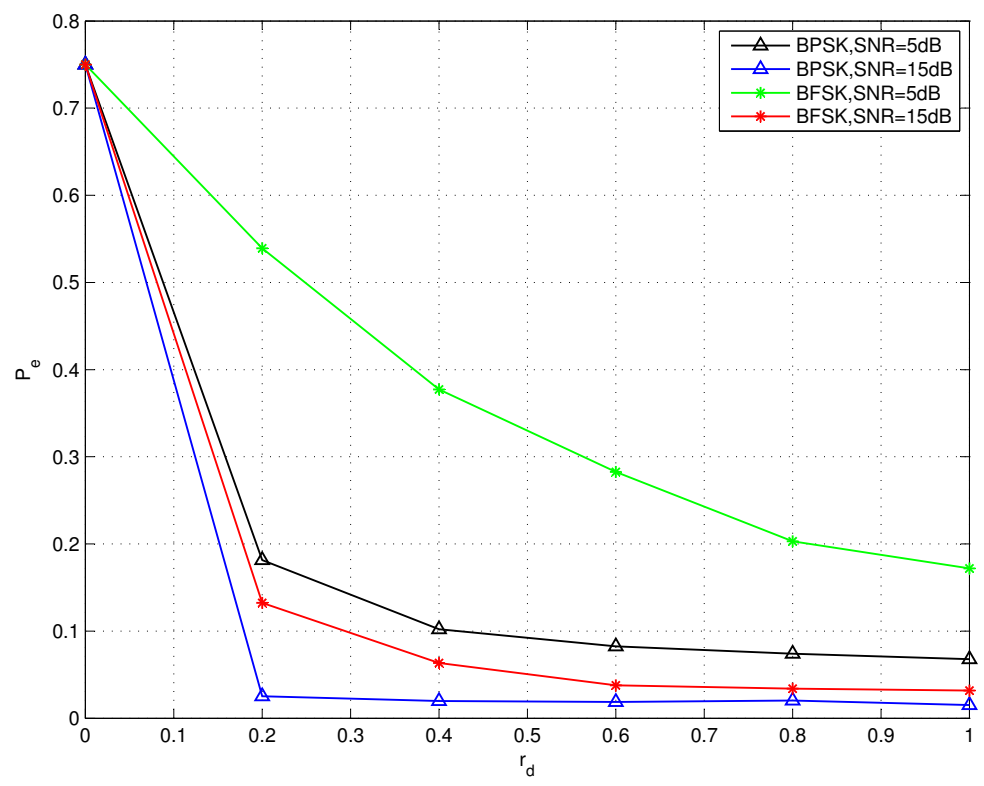

(b)

Figure 4.4: Rician fading channel model with BPSK and BFSK modulation: (a) $P_{e}$ vs. SNR, (b) $P_{e}$ vs. $r_{d}$. 


\section{CHAPTER 5: CONCLUSIONS}

In summary, we considered a distributed detection wireless system that is tasked with solving an $M$-ary hypothesis testing problem. We studied the effect of wireless channel uncertainty, due

to channel estimation error, on the design and performance of this system, assuming the sum of transmit powers of training and data symbols is fixed. In particular, we provided the optimal fusion rules for training and non-training based systems. Our results show that the error probability of this system, when the sensors employ FSK modulation, is minimized when training symbol transmit power is zero. However, when the sensors employ PSK modulation along with coherent reception at the FC the error probability is minimized when the transmit power is equally distributed between training and data symbols. 


\section{LIST OF REFERENCES}

[1] Z. Hajibabaei, A. Sani, and A. Vosoughi, "M-ary distributed detection in the presence of channel estimation error," in 2014 48th Asilomar Conference on Signals, Systems and Computers, Nov 2014, pp. 2147-2151.

[2] Z. Hajibabaei and A. Vosoughi, "Impact of wireless channel uncertainty upon m-ary distributed detection systems," in 2014 IEEE 25th Annual International Symposium on Personal, Indoor, and Mobile Radio Communication (PIMRC), Sept 2014, pp. 692-696.

[3] A. Sani and A. Vosoughi, "Resource allocation optimization for distributed vector estimation with digital transmission," in Signals, Systems and Computers, 2014 48th Asilomar Conference on, Nov 2014, pp. 1463-1467.

[4] Alireza Sani and Azadeh Vosoughi, "Bandwidth and power constrained distributed vector estimation in wireless sensor networks," in Military Communications Conference, MILCOM 2015 - 2015 IEEE, Oct 2015, pp. 1164-1169.

[5] A. Sani and A. Vosoughi, "On quantizer design for distributed estimation in bandwidth constrained networks," in Vehicular Technology Conference (VTC Fall), 2015 IEEE 82nd, Sept 2015, pp. 1-2.

[6] M. Shirazi and A. Vosoughi, "Bayesian cramer-rao bound for distributed vector estimation with linear observation model," in 2014 IEEE 25th Annual International Symposium on Personal, Indoor, and Mobile Radio Communication (PIMRC), Sept 2014, pp. 712-716.

[7] M. Shirazi and A. Vosoughi, "Bayesian cramer-rao bound for distributed estimation of correlated data with non-linear observation model," in 2014 48th Asilomar Conference on Signals, Systems and Computers, Nov 2014, pp. 1484-1488. 
[8] A. Sani and A. Vosoughi, "On distributed vector estimation for power and bandwidth constrained wireless sensor networks," IEEE Transactions on Signal Processing, vol. PP, no. 99, pp. 1-1, 2016.

[9] M. H. Chaudhary and L. Vandendorpe, "Power constrained linear estimation in wireless sensor networks with correlated data and digital modulation," IEEE Transactions on Signal Processing, vol. 60, no. 2, pp. 570-584, Feb 2012.

[10] Ruixin Niu, Biao Chen, and P.K. Varshney, "Fusion of decisions transmitted over rayleigh fading channels in wireless sensor networks," Signal Processing, IEEE Transactions on, vol. 54, no. 3, pp. 1018-1027, March 2006.

[11] Xiao-Gang Wang and H. C. Shen, "Multiple hypotheses testing strategy for distributed multisensor systems," in Intelligent Robots and Systems, 2000. (IROS 2000). Proceedings. 2000 IEEE/RSJ International Conference on, 2000, vol. 2, pp. 1440-1445 vol.2.

[12] P. Willett, P. F. Swaszek, and R. S. Blum, “The good, bad and ugly: distributed detection of a known signal in dependent gaussian noise," IEEE Transactions on Signal Processing, vol. 48, no. 12, pp. 3266-3279, Dec 2000.

[13] Wuyan, Liming, and Yangwanhai, "Optimal distributed decision fusion in the sense of the neyman-pearson test," in Radar, 2001 CIE International Conference on, Proceedings, 2001, pp. $708-712$.

[14] Q. Yan and R. S. Blum, "Distributed signal detection under the neyman-pearson criterion," IEEE Transactions on Information Theory, vol. 47, no. 4, pp. 1368-1377, May 2001.

[15] Xin Zhang, H.V. Poor, and Mung Chiang, "Optimal power allocation for distributed detection over mimo channels in wireless sensor networks," Signal Processing, IEEE Transactions on, vol. 56, no. 9, pp. 4124-4140, Sept 2008. 
[16] M.K. Banavar, A.D. Smith, C. Tepedelenlioglu, and A. Spanias, "On the effectiveness of multiple antennas in distributed detection over fading macs," Wireless Communications, IEEE Transactions on, vol. 11, no. 5, pp. 1744-1752, May 2012.

[17] C. R. Berger, M. Guerriero, S. Zhou, and P. Willett, "Pac vs. mac for decentralized detection using noncoherent modulation," IEEE Transactions on Signal Processing, vol. 57, no. 9, pp. 3562-3575, Sept 2009.

[18] A. Vosoughi and Yupeng Jia, "How does channel estimation error affect average sum-rate in two-way amplify-and-forward relay networks?," Wireless Communications, IEEE Transactions on, vol. 11, no. 5, pp. 1676-1687, May 2012.

[19] G. Taricco and G. C. Student Member, "Optimum receiver design for correlated rician fading mimo channels with pilot-aided detection," IEEE Journal on Selected Areas in Communications, vol. 25, no. 7, pp. 1311-1321, September 2007.

[20] H.R. Ahmadi and A. Vosoughi, "Impact of wireless channel uncertainty upon distributed detection systems," Wireless Communications, IEEE Transactions on, vol. 12, no. 6, pp. 2566-2577, June 2013.

[21] H.R. Ahmadi and A. Vosoughi, "Impact of channel estimation error on decentralized detection in bandwidth constrained wireless sensor networks," in Military Communications Conference, 2008. MILCOM 2008. IEEE, Nov 2008, pp. 1-7.

[22] I. Nevat, G.W. Peters, and I.B. Collings, "Distributed detection in sensor networks over fading channels with multiple antennas at the fusion centre," Signal Processing, IEEE Transactions on, vol. 62, no. 3, pp. 671-683, Feb 2014. 
[23] Jayesh H. Kotecha, V. Ramachandran, and A.M. Sayeed, "Distributed multitarget classification in wireless sensor networks," Selected Areas in Communications, IEEE Journal on, vol. 23, no. 4, pp. 703-713, April 2005.

[24] N. Maleki and A. Vosoughi, "Channel-aware m-ary distributed detection: Optimal and suboptimal fusion rules," in Statistical Signal Processing Workshop (SSP), 2012 IEEE, Aug 2012, pp. 644-647.

[25] A. Jeremic, Kon Max Wong, and Bin Liu, "Optimal distributed detection of multiple hypotheses using blind algorithm," in Acoustics, Speech and Signal Processing, 2009. ICASSP 2009. IEEE International Conference on, April 2009, pp. 2241-2244.

[26] H. s. Kim and N. A. Goodman, "Power control strategy for distributed multiple-hypothesis detection," IEEE Transactions on Signal Processing, vol. 58, no. 7, pp. 3751-3764, July 2010. 